\title{
Protéger notre eau
}

Dernière mise à jour : 8 octobre 2018

Le célèbre océanographe Jacques Yves Cousteau a dit un jour : " Toute vie fait partie d'un système de relations complexes dans lequel chacun dépend des autres, leur prend et leur donne quelque chose, et vit avec eux ". Cette observation se vérifie en particulier dans l'eau et c'est pourquoi la gestion de l'eau douce et les écosystèmes marins sont intimement liés à presque tous les problèmes environnementaux et humanitaires.

Prenons l'exemple d'une ville côtière en développement, un centre urbain dynamique et animé comme ceux qui abriteront une grande partie de la population mondiale à l'avenir. La plupart de ses habitants vit de la pêche ou travaille au port, plate-forme d'échanges où le va-et-vient des navires perturbe les écosystèmes qui se trouvent sous la surface. La gestion de l'eau doit répondre aux besoins des touristes affluant sur les plus belles plages, mais surtout à ceux des locaux. Pour être efficace, elle doit orchestrer les interactions entre de nombreux secteurs indispensables à la vie quotidienne, comme le génie civil, l'énergie et l'assainissement. Et elle n'est pas sans retentir sur la justice sociale et la stabilité de la gouvernance de la ville elle-même, loin de là.

Bref, les enjeux liés à l'eau sont omniprésents et les problèmes touchant aujourd'hui les réseaux hydrographiques et l'environnement marin auront de graves incidences dans des domaines aussi variés que les échanges, l'agriculture et la croissance inclusive. Dès lors que la gestion de l'eau, des océans et des écosystèmes est enrayée, le développement humain est entravé lui aussi.

L'humanité est extrêmement dépendante de l'environnement marin. Le produit national brut (PNB) à l'échelle mondiale est assuré à 61 \% par ce milieu et la subsistance d'environ 500 millions de personnes est tributaire d'activités liées à la mer. Et à l'intérieur des terres aussi, une gestion de l'eau efficace est vitale pour que les sociétés soient stables, justes et résilientes. Pourtant, pas moins de 2.1 milliards de personnes dans le monde n'ont toujours pas accès à une eau saine et sûre à domicile.

Les pressions qui s'exercent sur les réseaux hydrographiques et l'environnement marin dont nous dépendons continuent de s'accroître. Les activités pétrolières et gazières, les mines en eaux profondes et la pollution liée aux plastiques font des ravages dans le milieu marin et ont dégradé plus de $60 \%$ des principaux écosystèmes de la planète. Les infrastructures hydrauliques sont mises à rude épreuve par l'augmentation en flèche de la population mondiale et des ouvrages 
souvent non durables ou insuffisamment respectueux de l'environnement. A l'heure où le changement climatique vient jeter de l'huile sur le feu, quelles sont les mesures qui pourraient nous aider à relever ces défis entrecroisés et nous amener à un équilibre plus durable avec l'environnement marin ?

Pour les océans, la première chose à faire serait de préserver des écosystèmes autrefois prospères. Les zones marines protégées font partie des moyens permettant d'enrayer la destruction des écosystèmes et de favoriser leur régénération. La biodiversité devrait être prise en compte systématiquement dans tous les domaines d'action des pouvoirs publics et son encadrement juridique être renforcé. Par ailleurs, des mesures fortes devraient être adoptées et appliquées pour diminuer la pollution, tout comme des dispositions visant plus généralement à limiter la consommation de plastique non recyclable.

En dehors de la réduction de la pollution, il faudrait consacrer des investissements importants à la construction d'infrastructures d'approvisionnement en eau et d'assainissement et à l'amélioration de celles qui existent, en privilégiant les solutions naturelles. Les nouvelles technologies peuvent être mises à profit pour qu'elles soient durables et plus respectueuses de l'environnement, et pour améliorer les méthodes de traitement des eaux.

Lorsqu'ils œuvrent à ces progrès, les décideurs doivent garder à l'esprit les incidences qu'ils ont sur la vie quotidienne partout dans le monde. La gestion de l'eau est un élément fondamental dans la construction d'une société résiliente et stable, où les conflits sont plus rares. Elle joue aussi énormément dans la justice sociale en raison de ses répercussions sur la pauvreté et l'égalité entre les sexes. Par exemple, en Afrique et en Asie, les femmes parcourent en moyenne 6 kilomètres par jour - du temps qu'elles pourraient consacrer à des études, à un travail ou à une autre activité.

Pour que la gouvernance soit efficace dans tous ces domaines interconnectés, il faut coordonner l'action des parties prenantes dans les secteurs publics, privés et sans but lucratif. Comme on l'a entendu lors de la Semaine mondiale de l'eau de 2018, les responsables publics peuvent inciter les entreprises à s'entendre sur des normes communes. Une action à tous les niveaux est d'autant plus nécessaire que l'eau et les écosystèmes ne dépendent pas des frontières administratives et politiques. Afin de répondre à l'urgence des problèmes liés à l'eau, avec le soin et la coordination qu'ils requièrent et méritent, les parties prenantes doivent rechercher ensemble des solutions communes.

Vu l'étendue de leurs ramifications, les problématiques liés à l'eau et aux écosystèmes ne doivent pas être relégués à l'arrière plan de l'action publique. Comme l'a souligné Amina J. Mohammed, vice-Secrétaire générale des Nations Unies, à la Semaine mondiale de l'eau, le sixième Objectif de développement durable (ODD), axé sur l'eau, est en fait « la station d'accueil » de tous les autres ODD - le socle du développement humain. 
CL'Observateur de l'OCDE T3 2018

\section{Références}

Nations Unies, Objectif de développement durable no 6, https:// sustainabledevelopment.un.org/sdg6

Conférence des Nations Unies sur le développement durable (2012), Déclaration de Rio sur l'océan, www.unesco.org/new/fileadmin/MULTIMEDIA/HQ/SC/pdf/ pdf_Rio_Ocean_Declaration_2012.pdf

OCDE (2018), Implementing the OECD Principles on Water Governance, Éditions OCDE, https://www.oecd-ilibrary.org/fr/environment/implementing-the-oecdprinciples-on-water-governance_9789264292659-en

OCDE (2018), Mainstreaming Biodiversity for Sustainable Development, Éditions OCDE, https://www.oecd-ilibrary.org/fr/environment/mainstreamingbiodiversity-for-sustainable-development_9789264303201-en

OCDE (2017), Marine Protected Areas: Economics, Management and Effective Policy Mixes, Éditions OCDE https://www.oecd-ilibrary.org/fr/environment/ marine-protected-areas 9789264276208-en

We Are Water Foundation (2018), "Water and sanitation for gender equality" https://www.wearewater.org/en/water-and-sanitation-for-genderequality_290351

Organisation mondiale de la santé (2017), Communiqué du 12 juillet 2017, www.who.int/news-room/detail/12-07-2017-2-1-billion-people-lack-safedrinking-water-at-home-more-than-twice-as-many-lack-safe-sanitation

Semaine mondiale de l'eau www.worldwaterweek.org/tag/2018/ 